Recebido em 07/04/2018. Aprovado em 25/05/2018. Avaliado pelo sistema caudedindpeer review Publicado conforme normas da ABNT. http://dx.doi.org/10.22279/navus.2019.v9n1.p63-73.762

\title{
Energia solar em organizações militares: uma análise da viabilidade econômico-financeira
}

\author{
Alberto Jorge de Souza Júnior \\ Bacharel em Ciências Contábeis. Universidade Federal de Santa Maria (UFSM) - Brasil. \\ albertosousaj@hotmail.com \\ Wanderlei José Ghilardi \\ Doutor em Desenvolvimento Regional. Universidade Federal de Santa Maria (UFSM) - Brasil. \\ ghilard@terra.com.br \\ Sérgio Rossi Madruga \\ Doutor em Administração. Universidade Federal de Santa Maria (UFSM) - Brasil. \\ smadruga@smail.ufsm.br \\ Samia Mercado Alvarenga Bacharel em Ciências Econômicas. Universidade Federal de Sergipe (UFS) - Brasil. \\ samia alvarenga@hotmail.com
}

\section{RESUMO}

Este trabalho apresenta a análise da viabilidade econômico-financeira na instalação de um sistema de energia solar fotovoltaica nas Organizações Militares do Exército Brasileiro localizadas na cidade de Santa Maria (RS). Dentre os objetivos do trabalho estão a análise dos indicadores obtidos pelos métodos de viabilidade de projetos e a estimativa de tempo de retorno do investimento. Para a aplicação da viabilidade levantou-se os valores com o gasto de energia elétrica em tais organizações nos meses de agosto de 2015 a julho de 2016, os métodos aplicados compreendem Paybacksimples, Payback descontado, taxa interna de retorno (TIR) e valor presente líquido (VPL). Nas projeções de fluxo de caixa e demonstrativos de resultado verificou-se que os investimentos são atraentes. Através da TIR e VPL foi possível constatar que os projetos terão retorno significativo. Com base no Payback simples as instalações se mostram viáveis. Já com o Payback descontado o projeto de menor valor inicial não se mostrou exequível, pois o tempo de retorno foi praticamente o mesmo do período considerado para investimento. Entretanto, levando em consideração a preservação, a proteção e a melhoria da qualidade ambiental, bem como Política de Gestão Ambiental do Exército Brasileiro, a implantação dos projetos é extremamente relevante.

Palavras-chave: Viabilidade econômico-financeira. Energia solar. Organizações militares.

\section{Solar energy in military organizations: analysis of economic-financial viability}

\begin{abstract}
This present study provides an analysis of the financial-economic viability in the installation of a photovoltaic solar energy system in a military organization in the Brazilian Army, in Santa Maria City, Rio Grande do Sul State. Among the aims of the study are the analysis of indicators obtained through the method of project viability and an estimate of time for return on investment. For the viability application, the figures for electric energy costs in this organizations in the period between August, 2015 and July, 2016 were analyzed. The following methods were ap plied: simple Payback, discounted Payback, internal rate of return (IRR); and net present value (NPV). In the cash flow projections and income statements, it was found that the investments are attractive. Through the IRR and NPV, it was possible to verify that the projects will have a significant return. Based on simple Payback the facilities proved viable. However, with the discounted Payback, the lower cost project proved itself unviable, because the return time was practically the same as that in the considered time of investment. Nevertheless, considering the preservation, the protection and the improvement of the environment quality and the Environmental Management Politics in the Brazilian Army, the implementation of the project is extremely relevant.
\end{abstract}

Keywords: Financial and economic viability. Solar energy. Military organization. 


\section{INTRODUÇÃO}

A sustentabilidade tem como fim a preservação do meio ambiente para não comprometer os recursos naturais das gerações futuras. Nessa perspectiva, a ISO 14001 (ABNT, 2006) define o conceito de Gestão Ambiental para o sistema de gestão global da organização como sendo a forma pela qual a organização gerencia suas atividades buscando a otimização do desempenho ambiental via planejamento. Nesse sentido, o Exército, através da sua Política de Gestão Ambiental (PGAEB), tem o dever de conservar o meio ambiente, incentivando o estudo e a pesquisa de tecnologia para uso de fontes de alternativas de energia.

Ao encontro disso está o potencial energético solar brasileiro substancialmente maior do que o dos países do primeiro mundo, configurando amplas possibilidades de aproveitamento de energia alternativa e limpa (FARRET, 2010). Entre as técnicas de aproveitamento da energia solar destaca-se o uso de painéis fotovoltaicos onde a energia luminosa é convertida diretamente em energia elétrica. Esta forma de geração de energia recebeu regulação recente no Brasil através da Resolução Normativa n. 482, publicada em abril de 2012, a qual estabelece as condições para o acesso e regulamenta o sistema de compensação de energia elétrica.

Em 2010, a produção de coletores solares cresceu $21,1 \%$ no país em relação ao ano anterior, conforme pesquisa realizada pelo Departamento de Aquecimento Solar (Dasol). Trigueiro (2012) ressalta que as produções acumuladas de painéis solares no Brasil, chegaram à marca surpreendente de 6,24 milhões de metros quadrados no ano de 2012. Dada à expansão dessa tecnologia, bem como a facilidade de acesso ao público em geral a energia solar pode vir a ser uma alternativa viável para suprir as necessidades energéticas presentes e futuras da sociedade local e global.

Ainda que a economia conseguida por meio desses coletores compense as aplicações financeiras de sua instalação, é importante salientar que, no caso da energia fotovoltaica, o usuário deixa de ser mero consumidor, passando a ser um autoprodutor de energia elétrica. 0 que é uma situação bastante diferenciada, e nos remete a viabilidade de projetos especialmente no âmbito das organizações governamentais cuja adoção dos painéis solares implica em fortes investimentos públicos.

Assim, questiona-se a viabilidade econômica e financeira da instalação de um projeto de geração de energia solar fotovoltaica em Organizações Militares (OMs) do Exército Brasileiro na cidade de Santa Maria/RS. No intuito de oferecer respostas a essa problemática o presente estudo aplica métodos de análise de viabilidade de projetos de modo a obter indicadores que permitam averiguar o tempo de retorno do investimento proposto.

O exame crítico da viabilidade para a implantação do sistema fotovoltaico reduz a incerteza em relação ao investimento tornando-se sumariamente importante diante de um quadro de problemas ambientais mundiais, como o crescente aquecimento global. Estudos dessa natureza foram realizados por Moura (2015) e Ribeiro (2012) e se mostraram relevantes para a elaboração de políticas públicas, em virtude de ofertar projeções do real retorno do uso de energia solar em residências e organizações. Ademais, os resultados do presente estudo são de grande valia uma vez que a cidade de Santa Maria representa o segundo maior contingente militar do país.

Além desta introdução, a segunda seção apresenta o embasamento teórico e presta informações com a finalidade de sustentar a análise dos resultados. Na sequência, a seção terceira descreve o método de pesquisa, detalhando os procedimentos metodológicos executados para atingir aos objetivos propostos. $\mathrm{Na}$ quarta seção são apresentados e discutidos os resultados. 0 estudo se encerra com as considerações finais contendo as conclusões alcançadas e as dificuldades encontradas no desenvolvimento da pesquisa, além de recomendações para o desenvolvimento de estudos futuros.

\section{VIABILIDADE ECONÔMICA E FINANCEIRA DE PROJETOS}

A energia solar é uma fonte natural totalmente renovável e infinita que não faz barulho e não polui (DUTRA et al.,2013). Por ser proveniente da luz e do calor do sol é a fonte energética mais abundante da terra. O Brasil destaca-se como um país privilegiado na oferta de energia solar cujo potencial gira em torno de 2.500 
MW (megawatt), o que é cinco vezes maior do que o dos Estados Unidos e substancialmente maior do que o potencial dos países do primeiro mundo, configurando amplas possibilidades de aproveitamento (FARRET, 2010). Entre os benefícios da utilização da energia solar destaca-se a redução das emissões de gases poluentes na atmosfera, como também a economia de energia elétrica.

Entretanto, os custos iniciais para instalação de projetos para captação de energia solar são bastante elevados o que configura um entreposto na sua aquisição especialmente para setor público, haja vista, o contexto de crise financeira que força a racionalização de recursos, requerendo previsões e análises da viabilidade econômico-financeira. Estudos acerca da viabilidade de projetos fornecem uma estimativa do valor adicionado ao negócio de uma companhia ou uma pessoa diante de uma oportunidade com seu retorno através do tempo. Hirschfeld (2009) explica que para existir a viabilidade os benefícios resultantes de um investimento devem ser maiores que os custos ap licados.

Para Famá e Bruni (2003, p. 90) o prazo de recuperação de um capital aplicado é uma das primeiras etapas do processo de construção das estimativas, pois, "ao estudar a viabilidade de um investimento, uma das principais premissas assumidas costuma dizer respeito ao prazo máximo tolerado para a recuperação do capital aplicado". Um dos métodos para mensurar o tempo de recuperação de um investimento é a aplicação do Payback, também denominado tempo de repagamento do investimento ou do empréstimo.

o Payback nada mais é do que a quantidade de períodos que se leva para recuperar uma aplicação, ou seja, o tempo que o investimento leva para zerar seu fluxo acumulado." (MOTTA et al., 2009, p. 147). Famá e Bruni (2003, p. 91) salientam que esta é uma forma simples, fácil e direta para estimar o prazo de retorno e que não se utiliza do custo de capital do investimento. "Para obter o Payback simples de um projeto de investimento, basta verificar o tempo necessário para que o saldo do investimento seja igual a zero".

Como não se utiliza do custo de capital, a soma do saldo de investimento é feita através dos valores nominais, para atestar a viabilidade de um projeto segundo Payback, este, deve ser menor que o prazo de recuperação do que foi investido. Quando o valor do Payback for igual ao prazo de recuperação haverá indiferença na aceitação ou não do projeto e, caso o Paybackseja maior que o prazo de recuperação o projeto é inviável e não deve ser aceito.

Cherobim, Lemes e Rigo (2017) abordam o Payback descontado como um método de análise, capaz de evidenciar o tempo necessário para recuperar o investimento inicial. Este método, de acordo com os autores, considera o valor do dinheiro no tempo, pois, utiliza uma taxa de desconto para verificar o número exato de períodos, em que o projeto recupera o valor inicial investido.

Normalmente, a taxa de desconto usada é a taxa mínima de atratividade, a qual é determinada pelo próprio investidor como parâmetro para remuneração de seu capital. Por ser um método bastante simples é recomendável que uso do Payback descontado na análise de investimentos seja complementado por outros indicadores, como o valor presente líquido ou taxa interna de retorno, expresso na Equação 1.

$$
V_{P L}=\sum_{n=1}^{n=N} \frac{F c_{t}}{(1+i)^{n}}
$$

Onde:

$\mathrm{V}_{\mathrm{PL}}$ : Valor Presente Líquido;

Fc: fluxo de caixa;

t: momento em que o fluxo de caixa ocorreu;

i: taxa de desconto (ou taxa mínima de atratividade);

n: período de tempo.

O valor presente líquido (VPL) é obtido por meio do somatório dos fluxos de caixas descontados para a data atual ou presente resultando na adição de todos os fluxos de caixa na data zero. Para investimentos que requerem um desembolso inicial na expectativa de recebimento de fluxos de caixa futuros, o VPL representa 
os recebimentos vindouros trazidos e somados na data zero, subtraídos do investimento inicial, sendo assim um valor presente líquido do investimento inicial.

Para Souza (2003, p. 74), o VPL corresponde "à diferença entre o valor presente das entradas líquidas de caixa associadas ao projeto e o investimento inicial necessário". O seu valor é determinado subtraindo-se do valor inicial de um projeto, o valor presente das entradas líquidas de caixa, descontadas a uma taxa igual ao custo do capital da empresa (CHEROBIM; LEMES; RIGO, 2017). Segundo Famá e Bruni (2003) análises a partir do VPL são importantes pois esse método considera todos os fluxos de caixa originários do projeto, além de incluir no cálculo o custo de capital. Ademais, outra vantagem do VPL é que embutido no custo de capital estão custos inerentes ao risco.

Dessa forma, o VPL facilita a tomada de decisões, uma vez que, orienta o administrador financeiro a maximizar a riqueza do acionista ou proprietário (CHEROBIM; LEMES; RIGO, 2017). Ressalta-se que o VPL é o método de análise de investimento em projetos mais utilizado por profissionais de finanças, pelo fato de permitir interpretar facilmente os resultados (ABREU FILHO et al., 2003).

Além do VPL, outra importante ferramenta é a taxa interna de retorno (TIR) de um investimento que nada mais é do que a taxa exigida de retorno, quando utilizada como taxa de desconto, resulta em VPL igual a zero (ABREU FILHO et al., 2003). Uma análise mais cuidadosa do valor presente líquido revela que, à medida que o custo de capital aumenta, menor é a soma dos fluxos de caixa na data zero (FAMÁ; BRUNI, 2003). Assim, a TIR, é útil para determinar uma única taxa de retorno que sintetiza os méritos de um projeto.

Essa taxa é dita interna, no sentido de que depende somente dos fluxos de caixa do investimento (CHEROBIM; LEMES; RIGO, 2017). Para avaliar propostas de investimento, por meio do cálculo da TIR, é necessário conhecer os montantes de dispêndio de capital e dos fluxos de caixa líquidos gerados pela decisão, onde a TIR representará a rentabilidade do projeto expressa em termos de taxa de juros, conforme Equação 2 (ASSAF NETO, 2003).

$$
0=\sum_{n=1}^{\mathrm{n}=\mathrm{N}} \frac{\mathrm{Fc}_{\mathrm{t}}}{(1+\mathrm{Tir})^{\mathrm{n}}}
$$

Onde:

Tir: Taxa interna de retorno;

$\mathrm{Fc}_{\mathrm{t}}$ : Fluxo de caixa líquido no momento $\mathrm{n}$;

n: duração do projeto.

Desta forma, a TIR caracteriza a taxa de remuneração do capital investido, pois, representa a taxa de juros para a qual o valor presente das entradas de caixa resultantes do projeto iguala o valor presente dos desembolsos do mesmo, sendo uma medida bastante utilizada no orçamento de capital. Além da TIR Lapponi (2005), considerada ainda a taxa requerida que representa o risco do projeto de investimento, ou seja, se refere ao destino dado ao capital da empresa. A taxa requerida é obtida a partir dos custos dos fundos da empresa na data da avaliação do projeto de investimento, e reflete o risco médio de todos os ativos da empresa (LAPPONI, 2005).

\section{MÉTODO}

No intuito de atingir os objetivos propostos o presente estudo faz uso de uma pesquisa exploratória de caráter quantitativo, visto que se aplica cálculos econômico-financeiros com a finalidade de verificar a viabilidade do investimento em questão. Para o levantamento dos dados procedeu-se uma pesquisa documental. Marconi e Lakatos (2011) explicam que tal pesquisa se baseia em documentos, escritos ou não, tais como arquivos públicos, arquivos privados e fontes estatísticas.

Nesse estudo, serão utilizados tanto documentos públicos, quanto documentos privados. Os dados utilizados são provenientes de fontes secundárias sendo os gastos com energia elétrica obtidos diretamente 
nas OMs, através do levantamento das contas de energia elétrica de doze meses, período compreendido entre agosto do ano 2015 e julho de 2016, já os dados referentes ao investimento para implantação do sistema fotovoltaico foi obtido através de uma pesquisa de mercado em empresas especializada na instalação de projetos de energia solar.

A população estudada compreende as OMs de Santa Maria-RS, em virtude de o município concentrar o segundo maior contingente militar do Brasil. Para compor a amostra foram selecionadas três OMs tendo como critério o gasto de energia elétrica, desse modo os projetos de energia solar foram estudados para a OMs de maior gasto com energia elétrica (Projeto 1), OMs com gasto intermediário (Projeto 2) e OMs com menor gasto de energia elétrica (Projeto 3). A Figura 1 faz uma síntese das ações e métodos utilizados para estudar a viabilidade econômico-financeira das instalações fotovoltaicas.

Figura 1 - Ações para o alcance dos objetivos

Sondagem de orçamento de um possível fornecedor.

Cálculos de payback simples e descontado, do valor presente líquido e da taxa interna de retorno.

Comparação do investimento do projeto com os resultantes do retorno do investimento.

Análise do tempo de retorno do investimento e benefícios viabilizados em uma OM.

Fonte: Elaborado pelos autores, 2018.

Diante dos resultados obtidos foram constituídos índices que ensejam a comparação com o que foi 0 investimento do projeto, determinando em última instância, a exequibilidade ou não da implantação do sistema de energia solar fotovoltaica. Na seção que segue apresentam-se os valores de projetos foto voltaicos levantados juntamente a uma empresa especializada em energia limpa. Em seguida são discutidos valores inerentes à viabilidade econômico-financeira. Por fim, são exibidos os indicadores obtidos e apresentado o tempo de retorno do investimento no projeto de captação de energia solar fotovoltaica.

\section{RESULTADOS E DISCUSSÕES}

A análise da viabilidade econômico-financeira de um projeto pode ser feita de duas formas. A primeira delas, de forma simplificada a partir da estimativa do tempo de retorno simples do capital investido no projeto de um sistema fotovoltaico para suprimento energético. A outra forma, também objeto deste estudo, investiga o retorno descontado, verificando o valor presente do dispêndio de capital através do passar do tempo. Antes de se aplicar os métodos de viabilidade de projetos, é necessário fazer o levantamento do valor mensal de economia que a OM terá na fatura de energia elétrica. Esse valor será usado como parâmetro mensal de fluxo de caixa, bem como verificar o orçamento para instalação.

Previamente foram captadas informações, tais como a quantidade de radiação solar e a perda de potência devido à inclinação, pois, em razão da variação de incidência do sol durante os meses do ano, a inclinação e a posição dos painéis são fundamentais, já que podem provocar diversas perdas através do seu dimensionamento. Para os projetos foi considerada uma inclinação dos painéis em relação ao plano horizontal de 25 graus. 
Como parâmetro de instalação, utilizaram-se os telhados das OMs, situadas no centro da cidade de Santa Maria - RS. Para a identificação dos Investimentos necessários para cada projeto foi solicitado orçamento de uma empresa especializada em captação de energia solar. Segundo a empresa Sonnem Energia, o valor total para a instalação dos Projetos 1, 2 e 3 são respectivamente $R \$ 1.890 .060,00, R \$ 753.300,00$ e $R \$ 17.550,00$. Nesses valores estão inclusos gastos com painéis, inversores, estruturas de fixação e instalação completa, conforme Tabela 1.

Tabela 1 - Composição dos Projetos 1, 2 e 3 com valor total de instalação.

\begin{tabular}{|c|c|c|c|}
\hline \multirow[t]{2}{*}{ Composição do Sistema Fotovoltaico } & \multicolumn{3}{|c|}{ Quantidade } \\
\hline & Projeto 1 & Projeto 2 & Projeto 3 \\
\hline Módulos (painéis) em silício - Marca JINCO SOLAR & 1378 & 547 & 9 \\
\hline $\begin{array}{l}\text { Inversores (Conversor CC/AA) - Fronius Eco - } 27 \\
\text { kW }\end{array}$ & 15 & 6 & 1 \\
\hline Estruturas de fixação (Suporte em alumínio) & 1378 & 547 & 9 \\
\hline Instalação completa do sistema fotovoltaico & 1 & 1 & 1 \\
\hline Valor total do projeto & $\mathrm{R} \$ 1.890 .060,00$ & $\mathrm{R} \$ 753.300,00$ & $\mathrm{R} \$ 17.550,00$ \\
\hline
\end{tabular}

Fonte: Elaborado pelos autores, 2018.

Através da análise da fatura observa-se que o valor do kWh pago para a fornecedora nos meses de setembro e outubro de 2017 foi de $R \$ 1,070630$, percebe-se ainda que o valor do kWh pago em horário de ponta, que ocorre no período de três horas consecutivas no dia definido pela concessionária, exceto sábados, domingos e feriados é $247 \%$ maior que o horário fora de ponta, corresponde às demais 21 horas do dia.

Assim, decidiu-se verificar a média entre o horário de ponta e fora de ponta, dentro das bandeiras tarifárias vigentes e dos consumos dos meses de setembro e outubro de 2017, juntamente com os valores totais pagos na fatura. Desta forma, obteve-se o valor médio pago de $R \$ 0,3802$ por kWh. Foram desconsiderados para o cálculo da economia projetada os valo res de ICMS, PIS/PASEP e COFINS (tributos estes que fazem parte das faturas de energia elétrica).

Tendo-se apresentado o valor médio de geração dos projetos fotovoltaicos e o valor pago por kWh, chegamos ao valor que as OMs irão economizar mensalmente. Para tanto, procede-se um cálculo simples em que se multiplica o valor base do custo pela média de geração de kWh do sistema, por conseguinte, o produto é multiplicado por doze. A Tabela 2 ilustra economia mensal e anual projetada após a instalação dos três projetos.

Tabela 2 - Economia na fatura de energia elétrica mensal projetada.

\begin{tabular}{llll}
\hline & Projeto 1 & Projeto 2 & Projeto 3 \\
\hline Custo do kWh & $\mathrm{R} \$ 0,3802$ & $\mathrm{R} \$ 0,3802$ & $\mathrm{R} \$ 0,3802$ \\
Demanda mensal & $48344,76 \mathrm{kWh}$ & $19190,56 \mathrm{kWh}$ & $343,82 \mathrm{kWh}$ \\
Economia mensal projetada (Retorno) & $\mathrm{R} \$ 18.380,68$ & $\mathrm{R} \$ 7.296,25$ & $\mathrm{R} \$ 130,72$ \\
Economia anual projetada (Retorno) & $\mathrm{R} \$ 220.568,13$ & $\mathrm{R} \$ 87.555,01$ & $\mathrm{R} \$ 1.568,64$ \\
\hline
\end{tabular}

Fonte: Elaborado pelos autores, 2018.

Ao se considerar um custo do $\mathrm{kWh}$ de $\mathrm{R} \$ 0,3802$ e a possibilidade de geração média mensal dos sistemas propostos nos projetos de 48344,76 kWh, 19190,56 kWh e 343,82 kWh, é possível economizar anualmente $R \$ 220.568,13, R \$ 87.555,01$ e $R \$ 1.568,64$ em moeda corrente, ou seja, mensalmente $R \$ 18.380,68$ no Projeto $1, R \$ 87.555,01$ no Projeto 2 e $R \$ 1.568,64$ no Projeto 3.

Com os valores totais orçados e a economia total anual nas faturas calcula-se o Payback simples e Payback descontado, juntamente com a aplicação do valor presente líquido (VPL) e da taxa interna de retorno (TIR). Tendo em vista que o tempo de vida útil de um sistema fotovoltaico é de 25 anos, utilizou-se esse valor como parâmetro para o tempo de retorno do investimento. Para taxa requerida (utilizada como taxa mínima de atratividade - TMA) aplicada aos cálculos adotou-se o percentual de 7,4\% ao ano, que foi definida a partir 
da Taxa Selic. Efetuou-se a pesquisa do valor desta taxa, no Banco Central do Brasil, referente ao dia 6 de novembro de 2017 (BCB, 2017b). Os resultados dos cálculos do Payback para os Projetos 1, 2 e 3 são demonstrados, respectivamente, nas Tabelas 3,4 e 5.

Tabela 3 - Cálculo do Paybacksimples e Payback descontado para o Projeto1.

\begin{tabular}{|c|c|c|c|c|c|}
\hline \multicolumn{2}{|c|}{ Investimento - R\$ 1.890.060,00 } & \multicolumn{3}{|c|}{ Taxa Requerida (TMA) - Ao ano - 7,4\% a.a. } & \multirow[b]{2}{*}{ Ano } \\
\hline Fluxo de caixa & PaybackSimples & $\begin{array}{l}\text { Fluxo de } \\
\text { Descontado }\end{array}$ & caixa & Payback Descontado & \\
\hline & $-R \$ 1.890 .060,00$ & & & $-R \$ 1.890 .060,00$ & 0 \\
\hline$R \$ 220.568,13$ & $-R \$ 1.669 .491,87$ & $\mathrm{R} \$ 205.370,70$ & & $-R \$ 1.684 .689,30$ & 1 \\
\hline$R \$ 220.568,13$ & $-R \$ 1.448 .923,74$ & $\mathrm{R} \$ 191.220,39$ & & -R\$ 1.493.468,91 & 2 \\
\hline$R \$ 220.568,13$ & $-R \$ 1.228 .355,61$ & $\mathrm{R} \$ 178.045,06$ & & $-R \$ 1.315 .423,85$ & 3 \\
\hline$R \$ 220.568,13$ & $-R \$ 1.007 .787,48$ & $\mathrm{R} \$ 165.777,52$ & & -R\$ 1.149.646,33 & 4 \\
\hline$R \$ 220.568,13$ & $-R \$ 787.219,35$ & $R \$ 154.355,23$ & & -R\$ 995.291,10 & 5 \\
\hline$R \$ 220.568,13$ & $-R \$ 566.651,22$ & $\mathrm{R} \$ 143.719,96$ & & $-R \$ 851.571,14$ & 6 \\
\hline$R \$ 220.568,13$ & $-R \$ 346.083,09$ & $\mathrm{R} \$ 133.817,46$ & & $-R \$ 717.753,68$ & 7 \\
\hline$R \$ 220.568,13$ & $-R \$ 125.514,96$ & $\mathrm{R} \$ 124.597,27$ & & -R\$ 593.156,41 & 8 \\
\hline$R \$ 220.568,13$ & $\mathrm{R} \$ 95.053,17$ & $\mathrm{R} \$ 116.012,35$ & & $-\mathrm{R} \$ 477.144,06$ & 9 \\
\hline$R \$ 220.568,13$ & $\mathrm{R} \$ 315.621,30$ & $\mathrm{R} \$ 108.018,95$ & & $-R \$ 369.125,11$ & 10 \\
\hline $\mathrm{R} \$ 220.568,13$ & $\mathrm{R} \$ 536.189,43$ & $\mathrm{R} \$ 100.576,30$ & & $-R \$ 268.548,81$ & 11 \\
\hline$R \$ 220.568,13$ & $\mathrm{R} \$ 756.757,56$ & $R \$ 93.646,46$ & & $-R \$ 174.902,35$ & 12 \\
\hline$R \$ 220.568,13$ & $\mathrm{R} \$ 977.325,69$ & $\mathrm{R} \$ 87.194,10$ & & $-R \$ 87.708,25$ & 13 \\
\hline$R \$ 220.568,13$ & $\mathrm{R} \$ 1.197 .893,82$ & $\mathrm{R} \$ 81.186,31$ & & $-R \$ 6.521,94$ & 14 \\
\hline$R \$ 220.568,13$ & $\mathrm{R} \$ 1.418 .461,95$ & $\mathrm{R} \$ 75.592,47$ & & $R \$ 69.070,53$ & 15 \\
\hline$R \$ 220.568,13$ & $\mathrm{R} \$ 1.639 .030,08$ & $\mathrm{R} \$ 70.384,05$ & & $\mathrm{R} \$ 139.454,58$ & 16 \\
\hline $\mathrm{R} \$ 220.568,13$ & $\mathrm{R} \$ 1.859 .598,21$ & $\mathrm{R} \$ 65.534,50$ & & $\mathrm{R} \$ 204.989,08$ & 17 \\
\hline$R \$ 220.568,13$ & $\mathrm{R} \$ 2.080 .166,34$ & $\mathrm{R} \$ 61.019,09$ & & $R \$ 266.008,17$ & 18 \\
\hline$R \$ 220.568,13$ & $\mathrm{R} \$ 2.300 .734,47$ & $\mathrm{R} \$ 56.814,79$ & & $\mathrm{R} \$ 322.822,96$ & 19 \\
\hline$R \$ 220.568,13$ & $\mathrm{R} \$ 2.521 .302,60$ & $R \$ 52.900,18$ & & $\mathrm{R} \$ 375.723,14$ & 20 \\
\hline$R \$ 220.568,13$ & $\mathrm{R} \$ 2.741 .870,73$ & $R \$ 49.255,29$ & & $\mathrm{R} \$ 424.978,43$ & 21 \\
\hline$R \$ 220.568,13$ & $\mathrm{R} \$ 2.962 .438,86$ & $\mathrm{R} \$ 45.861,53$ & & $\mathrm{R} \$ 470.839,96$ & 22 \\
\hline$R \$ 220.568,13$ & $\mathrm{R} \$ 3.183 .006,99$ & $\mathrm{R} \$ 42.701,61$ & & $\mathrm{R} \$ 513.541,57$ & 23 \\
\hline$R \$ 220.568,13$ & $\mathrm{R} \$ 3.403 .575,12$ & $R \$ 39.759,42$ & & $R \$ 553.300,99$ & 24 \\
\hline$R \$ 220.568,13$ & $\mathrm{R} \$ 3.624 .143,25$ & $R \$ 37.019,94$ & & $R \$ 590.320,93$ & 25 \\
\hline \multirow[t]{2}{*}{ TIR } & $10,76 \%$ & VPL & & $\mathrm{R} \$ 590.320,93$ & \\
\hline & Ano & Meses & & Dias & \\
\hline Payback Simples & 8 & 6 & & 25 & \\
\hline Payback Descontado & 14 & 1 & & 1 & \\
\hline
\end{tabular}

Fonte: Elaborado pelos autores, 2018.

Os resultados da Tabela 3 demonstram, que através do cálculo do Paybacksimples, são necessários 8 anos, 6 meses e 25 dias para recuperar o investimento inicial proposto de $R \$ 1.890 .060,00$, já, segundo o cálculo do Payback descontado, esse mesmo investimento precisaria de 14 anos, 1 mês e 1 dia para obter retorno. Nota-se que o valor presente líquido ao final do vigésimo quinto ano será de R\$590.320,93 e a taxa interna de retorno de 10,76\%. Observa-se, ainda, que apenas com a economia acumulada durante um período de 9 anos ( $R \$ 220.568,13,45 \times 9$ ), é possível identificar um valor total de $R \$ 1.985 .113,17$, ou seja, é $R \$ 95.053,17$ superior ao custo total da instalação do sistema de geração solar.

Portanto, a partir de, aproximadamente, 8 anos, 6 meses e 25 dias o sistema já estará totalmente pago e gerando uma economia anual de $R \$ 220.568,13$. Após os 25 anos analisados, em valores totais, o Payback simples mostra um retorno de até $R \$ 3.624 .143,00$ e o Payback descontado de $R \$ 590.320,93$ evidenciando que ao final do tempo utilizado como parâmetro o investimento cobre também os custos de manutenção, que se faz por volta de 10 a $15 \%$ do valor inicial ( $R \$ 189.006,00$ a R $\$ 283.509,00$ ). A partir da TIR de $10,76 \%$, estimase que este projeto renda 3,36\% acima da Taxa Selic o que sugere a viabilidade do Projeto 1. Na sequência, a Tabela 4 mostra os resultados para Projeto 2, cujo investimento inicial é de $\mathrm{R} \$ 753.300,00$. 
Tabela 4 - Cálculo do Paybacksimples e Payback descontado para o Projeto2.

\begin{tabular}{|c|c|c|c|c|}
\hline \multicolumn{2}{|c|}{ Investimento - R\$753.300,00 } & \multicolumn{2}{|c|}{ Taxa Requerida (TMA) - Ao ano - 7,4\% a.a. } & \multirow[b]{2}{*}{ Ano } \\
\hline Fluxo de caixa & PaybackSimples & $\begin{array}{lll}\text { Fluxo de } & \text { caixa } \\
\text { Descontado } & \\
\end{array}$ & Payback Descontado & \\
\hline & $-R \$ 753.300,00$ & & $-R \$ 753.300,00$ & 0 \\
\hline$R \$ 87.555,01$ & $-R \$ 665.744,99$ & $R \$ 81.517,70$ & $-R \$ 671.782,30$ & 1 \\
\hline$R \$ 87.555,01$ & $-R \$ 578.189,98$ & $\mathrm{R} \$ 75.901,02$ & $-R \$ 595.881,28$ & 2 \\
\hline $\mathrm{R} \$ 87.555,01$ & $-R \$ 490.634,97$ & $R \$ 70.671,34$ & $-R \$ 525.209,94$ & 3 \\
\hline$R \$ 87.555,01$ & $-R \$ 403.079,96$ & $R \$ 65.802,00$ & $-R \$ 459.407,94$ & 4 \\
\hline$R \$ 87.555,01$ & $-R \$ 315.524,95$ & $R \$ 61.268,05$ & $-R \$ 398.139,89$ & 5 \\
\hline $\mathrm{R} \$ 87.555,01$ & $-R \$ 227.969,94$ & $\mathrm{R} \$ 57.046,70$ & $-R \$ 341.093,19$ & 6 \\
\hline $\mathrm{R} \$ 87.555,01$ & $-R \$ 140.414,93$ & $R \$ 53.116,11$ & $-R \$ 287.977,08$ & 7 \\
\hline $\mathrm{R} \$ 87.555,01$ & $-R \$ 52.859,92$ & $R \$ 49.456,34$ & $-R \$ 238.520,74$ & 8 \\
\hline $\mathrm{R} \$ 87.555,01$ & $\mathrm{R} \$ 34.695,09$ & $R \$ 46.048,73$ & $-\mathrm{R} \$ 192.472,01$ & 9 \\
\hline $\mathrm{R} \$ 87.555,01$ & $\mathrm{R} \$ 122.250,10$ & $R \$ 42.875,91$ & -R\$ 149.596,10 & 10 \\
\hline$R \$ 87.555,01$ & $\mathrm{R} \$ 209.805,11$ & $R \$ 39.921,71$ & -R\$ 109.674,39 & 11 \\
\hline $\mathrm{R} \$ 87.555,01$ & $\mathrm{R} \$ 297.360,12$ & $\mathrm{R} \$ 37.171,05$ & $-R \$ 72.503,34$ & 12 \\
\hline $\mathrm{R} \$ 87.555,01$ & $\mathrm{R} \$ 384.915,13$ & $R \$ 34.609,92$ & $-R \$ 37.893,42$ & 13 \\
\hline $\mathrm{R} \$ 87.555,01$ & $\mathrm{R} \$ 472.470,14$ & $R \$ 32.225,25$ & $-R \$ 5.668,17$ & 14 \\
\hline $\mathrm{R} \$ 87.555,01$ & $\mathrm{R} \$ 560.025,15$ & $R \$ 30.004,89$ & $R \$ 24.336,72$ & 15 \\
\hline $\mathrm{R} \$ 87.555,01$ & $\mathrm{R} \$ 647.580,16$ & $R \$ 27.937,51$ & $\mathrm{R} \$ 52.274,23$ & 16 \\
\hline$R \$ 87.555,01$ & $\mathrm{R} \$ 735.135,17$ & $R \$ 26.012,58$ & $R \$ 78.286,81$ & 17 \\
\hline$R \$ 87.555,01$ & $\mathrm{R} \$ 822.690,18$ & $R \$ 24.220,28$ & $\mathrm{R} \$ 102.507,09$ & 18 \\
\hline $\mathrm{R} \$ 87.555,01$ & $\mathrm{R} \$ 910.245,19$ & $\mathrm{R} \$ 22.551,47$ & $\mathrm{R} \$ 125.058,56$ & 19 \\
\hline$R \$ 87.555,01$ & $\mathrm{R} \$ 997.800,20$ & $R \$ 20.997,64$ & $\mathrm{R} \$ 146.056,20$ & 20 \\
\hline $\mathrm{R} \$ 87.555,01$ & $\mathrm{R} \$ 1.085 .355,21$ & $R \$ 19.550,88$ & $\mathrm{R} \$ 165.607,08$ & 21 \\
\hline $\mathrm{R} \$ 87.555,01$ & $\mathrm{R} \$ 1.172 .910,22$ & $R \$ 18.203,80$ & $\mathrm{R} \$ 183.810,88$ & 22 \\
\hline $\mathrm{R} \$ 87.555,01$ & $\mathrm{R} \$ 1.260 .465,23$ & $R \$ 16.949,53$ & $\mathrm{R} \$ 200.760,41$ & 23 \\
\hline$R \$ 87.555,01$ & $\mathrm{R} \$ 1.348 .020,24$ & $R \$ 15.781,69$ & $\mathrm{R} \$ 216.542,10$ & 24 \\
\hline $\mathrm{R} \$ 87.555,01$ & $\mathrm{R} \$ 1.435 .575,25$ & $\mathrm{R} \$ 14.694,31$ & $\mathrm{R} \$ 231.236,41$ & 25 \\
\hline \multirow[t]{2}{*}{ TIR } & $10,71 \%$ & VPL & $\mathrm{R} \$ 231.236,41$ & \\
\hline & Ano & Meses & Dias & \\
\hline Payback Simples & 8 & 7 & 7 & \\
\hline Payback Descontado & 14 & 2 & 8 & \\
\hline
\end{tabular}

Semelhante ao Projeto 1, com TIR encontrada de 10,71\%, o Projeto 2 se torna viável para o período analisado, pois mostra ser 3,31\% mais rentável que a Taxa Selic e com VPL ao final do vigésimo quinto ano de R\$231.236,41. Através do cálculo do Payback simples são necessários 8 anos, 7 meses e 7 dias e do cálculo do Payback descontado são necessários 14 anos, 2 meses e 8 dias para recuperar o investimento inicial proposto de $R \$ 753.300,00$.

A economia acumulada durante um período de 9 anos ( $R \$ 87.555,01 \times 9$ ) identifica um valor total de $R \$ 787.995,09$, ou seja, $R \$ 34.695,09$ superior ao custo total da instalação do sistema de geração solar. Portanto, a partir desse perío do o Projeto 2 também estará totalmente pago e gerando $\mathrm{R} \$ 87.555,01$ de economia anual em energia elétrica. Em valores totais, os Paybacks simples e descontado mostram um retorno de até $R \$$ $1.435 .575,25$ e de $R \$ 234.236,41$, respectivamente após os 25 anos de vida útil do sistema. Isso remete a cobertura dos custos de manutenção no final do período analisado, que se faz entre $R \$ 75.330,00$ e $R \$$ $112.995,00$ (10 a 15\%) do valor inicial do investimento. Por fim, a Tabela 5 evidencia os resultados para o Projeto 3 que mostra os cálculos para o investimento de $\mathrm{R} \$ 17.550,00$. 
Tabela 5 - Cálculo do Payback simples e Payback descontado para o Projeto 3

\begin{tabular}{|c|c|c|c|c|c|}
\hline \multicolumn{2}{|c|}{ Investimento - R\$ $17.550,00$} & \multicolumn{3}{|c|}{ Taxa Requerida (TMA) - Ao ano - 7,4\% a.a. } & \multirow[b]{2}{*}{ Ano } \\
\hline Fluxo de caixa & PaybackSimples & $\begin{array}{l}\text { Fluxo de } \\
\text { Descontado }\end{array}$ & caixa & Payback Descontado & \\
\hline & $-\mathrm{R} \$ 17.550,00$ & & & $-R \$ 17.550,00$ & 0 \\
\hline $\mathrm{R} \$ 1.568,64$ & $-\mathrm{R} \$ 15.981,36$ & $\mathrm{R} \$ 1.460,56$ & & $-R \$ 16.089,44$ & 1 \\
\hline $\mathrm{R} \$ 1.568,64$ & $-\mathrm{R} \$ 14.412,72$ & $\mathrm{R} \$ 1.359,92$ & & $-R \$ 14.729,52$ & 2 \\
\hline $\mathrm{R} \$ 1.568,64$ & $-\mathrm{R} \$ 12.844,08$ & $\mathrm{R} \$ 1.266,22$ & & $-R \$ 13.463,30$ & 3 \\
\hline $\mathrm{R} \$ 1.568,64$ & $-\mathrm{R} \$ 11.275,44$ & $\mathrm{R} \$ 1.178,98$ & & $-\mathrm{R} \$ 12.284,32$ & 4 \\
\hline $\mathrm{R} \$ 1.568,64$ & $-R \$ 9.706,80$ & $\mathrm{R} \$ 1.097,75$ & & $-\mathrm{R} \$ 11.186,57$ & 5 \\
\hline $\mathrm{R} \$ 1.568,64$ & $-R \$ 8.138,16$ & $\mathrm{R} \$ 1.022,11$ & & $-\mathrm{R} \$ 10.164,46$ & 6 \\
\hline $\mathrm{R} \$ 1.568,64$ & $-R \$ 6.569,52$ & $\mathrm{R} \$ 951,69$ & & $-R \$ 9.212,77$ & 7 \\
\hline $\mathrm{R} \$ 1.568,64$ & $-R \$ 5.000,88$ & $\mathrm{R} \$ 886,11$ & & -R\$ 8.326,66 & 8 \\
\hline$R \$ 1.568,64$ & $-R \$ 3.432,24$ & $R \$ 825,06$ & & $-R \$ 7.501,60$ & 9 \\
\hline $\mathrm{R} \$ 1.568,64$ & $-R \$ 1.863,60$ & $\mathrm{R} \$ 768,21$ & & -R\$ 6.733,39 & 10 \\
\hline $\mathrm{R} \$ 1.568,64$ & $-R \$ 294,96$ & $\mathrm{R} \$ 715,28$ & & $-R \$ 6.018,11$ & 11 \\
\hline $\mathrm{R} \$ 1.568,64$ & $\mathrm{R} \$ 1.273,68$ & $\mathrm{R} \$ 666,00$ & & $-R \$ 5.352,11$ & 12 \\
\hline $\mathrm{R} \$ 1.568,64$ & $\mathrm{R} \$ 2.842,32$ & $\mathrm{R} \$ 620,11$ & & $-R \$ 4.732,00$ & 13 \\
\hline$R \$ 1.568,64$ & $R \$ 4.410,96$ & $\mathrm{R} \$ 577,38$ & & $-R \$ 4.154,62$ & 14 \\
\hline$R \$ 1.568,64$ & $R \$ 5.979,60$ & $R \$ 537,60$ & & $-R \$ 3.617,02$ & 15 \\
\hline$R \$ 1.568,64$ & $R \$ 7.548,24$ & $R \$ 500,56$ & & $-R \$ 3.116,46$ & 16 \\
\hline $\mathrm{R} \$ 1.568,64$ & $\mathrm{R} \$ 9.116,88$ & $\mathrm{R} \$ 466,07$ & & $-R \$ 2.650,39$ & 17 \\
\hline $\mathrm{R} \$ 1.568,64$ & $\mathrm{R} \$ 10.685,52$ & $R \$ 433,96$ & & $-R \$ 2.216,43$ & 18 \\
\hline $\mathrm{R} \$ 1.568,64$ & $\mathrm{R} \$ 12.254,16$ & $R \$ 404,06$ & & $-\mathrm{R} \$ 1.812,37$ & 19 \\
\hline$R \$ 1.568,64$ & $\mathrm{R} \$ 13.822,80$ & $R \$ 376,22$ & & $-R \$ 1.436,15$ & 20 \\
\hline $\mathrm{R} \$ 1.568,64$ & $\mathrm{R} \$ 15.391,44$ & $R \$ 350,29$ & & $-R \$ 1.085,86$ & 21 \\
\hline $\mathrm{R} \$ 1.568,64$ & $\mathrm{R} \$ 16.960,08$ & $R \$ 326,16$ & & $-R \$ 759,70$ & 22 \\
\hline $\mathrm{R} \$ 1.568,64$ & $\mathrm{R} \$ 18.528,72$ & $R \$ 303,69$ & & $-R \$ 456,01$ & 23 \\
\hline $\mathrm{R} \$ 1.568,64$ & $\mathrm{R} \$ 20.097,36$ & $\mathrm{R} \$ 282,76$ & & $-R \$ 173,25$ & 24 \\
\hline $\mathrm{R} \$ 1.568,64$ & $\mathrm{R} \$ 21.666,00$ & $\mathrm{R} \$ 263,28$ & & $\mathrm{R} \$ 90,03$ & 25 \\
\hline \multirow[t]{2}{*}{ TIR } & $7,46 \%$ & VPL & & $\mathrm{R} \$ 90,03$ & \\
\hline & Anos & Meses & & Dias & \\
\hline Payback Simples & 11 & 2 & & 8 & \\
\hline Payback Descontado & 24 & 7 & & 27 & \\
\hline
\end{tabular}

Fonte: Elaborado pelos autores, 2018.

Nos cálculos referentes ao Projeto 3, foi obtida uma TIR de 7,46\%, se mostrando 0,06\% mais rentável que a Taxa Selic, entretanto essa é uma margem apertada, tornando-se pouco viável para o período em análise. Por meio do Payback descontado verifica-se que são necessários 24 anos, 7 meses e 27 dias para recuperar 0 investimento inicial proposto de R\$17.550,00 e O VPL ao final do vigésimo quinto ano é de apenas R\$ 90,03. Apesar de ser positivo, não cobre os valores da manutenção prevista para o tempo de vida útil, que gira entre $\mathrm{R} \$ 1.755,00$ e $\mathrm{R} \$ 2.632,50$, mostrando que provavelmente deverá haver maior dispêndio de recursos para este fim.

Através do cálculo do Paybacksimples são necessários 11 anos, 2 meses e 8 dias, situação diferenciada em se tratando de retorno simples, mostrando-se viável a instalação do projeto. A economia acumulada durante um período de 12 anos ( $R \$ 1.568,64 \times 12$ ) identifica um valor total de $R \$ 18.823,68$, ou seja, $R \$ 1.273,68$ superior ao custo total da instalação do sistema de geração solar. Portanto, a partir de, aproximadamente, 11 anos, 2 meses e 8 dias o sistema já estará totalmente pago e gerando uma economia anual de $R \$ 1.568,64$, assim, cobre-se os valores de manutenção. Em valores totais, o Paybacksimples mostra um retorno de até $\mathrm{R} \$$ $21.666,00$ após os 25 anos de vida útil do sistema. 


\section{CONSIDERAÇÕES FINAIS}

O presente trabalho teve como escopo identificar a viabilidade econômico-financeira na implantação de energia solar fotovoltaica em OMs. Para isso, foram colhidos dados para subsidiar a montagem de fluxos de caixa, buscando-se informações junto às OMs e em empresa especializada no ramo de energia solar. Dentro dos objetivos específicos, foi possível expor a preocupação com o consumo e sustentabilidade pelo poder público e valores dos orçamentos de projetos de implantação de energia fotovoltaica. Com isso, foram aplicados os métodos de viabilidade econômica, analisando os indicadores e estimando o tempo de retorno do investimento.

Para os cálculos e análises de viabilidade de projeto têm-se os métodos de Paybacksimples e Payback descontado e retorno do investimento em energia fotovoltaica os quais permitiram uma avaliação econômica e financeira entre o investimento realizado com a economia projetada, ou seja, a redução de recursos públicos aplicados em energia elétrica. Nas projeções de fluxo de caixa e demonstrativos de resultado, verificou-se que os investimentos são atraentes. Através da TIR e VPL foi possível constatar que para os três cenários esperados, os projetos terão retorno significativo e se mostram bastante viáveis, se tratando de retorno simples, ou seja, através do Payback simples. Assim, considerando o tempo de retorno simples e a vida útil média do sistema fotovoltaico pode-se dizer que estes sistemas são economicamente vantajosos.

No entanto, para a análise via Payback descontados, que leva em consideração a desvalorização da moeda através do tempo, um dos projetos, o projeto de menor valor, se mostrou inviável do ponto de vista financeiro, pois o tempo de retorno através do fluxo descontado foi praticamente o mesmo do período considerado para investimento. A despeito disso, os outros do is projetos deram sinais de que o investimento é perfeitamente exequível, trazendo grandes benefícios para as OMs em termos de economia de recursos financeiros.

Um ponto a ser considerado é o elevado custo inicial na instalação do projeto fotovoltaico, que se torna um entreposto comercial para a aquisição nesta forma de geração de energia elétrica, especialmente para o setor público, haja vista a crise financeira que força a racionalização de recursos, devendo haver previsão para a disponibilização desses numerários juntamente com a reorganização da dotação orçamentária pelo Exército Brasileiro.

Em suma, o estudo foi alicerçado na preocupação com a política ambiental e estruturado de modo a desmistificar o emprego da energia renovável, que, não raro, é atrelada a custos altos de instalação. Os resultados dessa pesquisa demonstram a possibilidade de redução de gastos de recursos públicos, bem como a melhoria no aproveitamento e na diversidade energética associadas à preservação ambiental incentivando o uso de energia solar como uma alternativa limpa à energia elétrica.

\section{REFERÊNCIAS}

ABREU FILHO, J. F. de et al. Finanças corporativas. Rio de Janeiro: FGV, 2003.

ASSAF NETO, A. Finanças corporativas e valor. São Paulo: Atlas, 2003.

ASSOCIAÇÃO BRASILEIRA DE NORMAS TÉCNICAS (ABNT). ISO 14001: Sistema de Gestão Ambiental (SGA): especificações para implantação e guia. Rio de J aneiro: ABNT, 2006.

CHEROBIM, Ana Paula; LEMES, Antonio; RIGO, Claudio. Administração financeira: princípios, fundamentos e práticas brasileiras. São Paulo: Elsevier Brasil, 2017.

DUTRA, J . C. D. N. et al. Uma análise do panorama das regiões missões e noroeste do Estado do Rio Grande do Sul sob o prisma da energia eólica e solar fotovoltaica como fontes alternativas de energia. Revista

Paranaense de Desenvolvimento, v. 34, n. 124, p. 225-243, 2013.

FAMÁ, Rubens; BRUNI, Adriano Leal. As decisões de Investimentos. São Paulo: Atlas, 2003. 
FARRET, F. A. Aproveitamento de pequenas fontes de energia elétrica. 2. ed. Santa Maria: UFSM, 2010.

HIRSCHFELD, H. Engenharia econômica e análise de custos: aplicações práticas para economistas, engenheiros, analistas de investimentos e administradores. 7. ed. São Paulo: Atlas, 2009.

LAPPONI, J. C. Matemática financeira: redesenho organizacional para o crescimento e desempenho máximos. Rio de Janeiro: Elsevier, 2005.

MARCONI, M. de A.; LAKATOS, E. M. Técnicas de pesquisa. 7. ed. São Paulo: Atlas, 2011.

MOURA, G. D. et al. Análise da viabilidade econômico-financeira da energia solar fotovoltaica em uma Instituição de Ensino Superior do Sul do Brasil. In: CONGRESSO BRASILEIRO DE CUSTOS, 22., 2015, Foz do Iguaçu. Anais eletrônicos... Foz do Iguaçu - PR: CBC, 2015. Disponível em:

https://edisciplinas.usp.br/pluginfile.php/3445027/mod_resource/content/1/solar\%20complemento.pdf. Acesso em: 16 jun. 2017.

MOTTA, R. da R. et al. Engenharia econômica e finanças. Rio de Janeiro: Elsevier, 2009.

RIBEIRO, U. G. V. Estudo de viabilidade econômica de instalação de fontes de energia renováveis baseadas em células fotovoltaicas para o uso residencial. 2012. 58 f. Monografia (Graduação em Engenharia Elétrica com ênfase em Sistemas de Energia e Automação) - Escola de Engenharia de São Carlos, Universidade de São Paulo, São Paulo, 2012. Disponível em: http://www.tcc.sc.usp.br/tce/disponiveis/18/180500/tce-18042013- 145705/?\&lang=br. Acesso em: 11 nov. 2017.

SOUZA, A. B. Projetos de investimentos de capital: elaboração, análise, tomada de decisão. São Paulo: Atlas, 2003.

TRIGUEIRO, A. Mundo sustentável 2: novos rumos para um planeta em crise. São Paulo: Globo, 2012. 